\title{
Referee Acknowledgement for 2012
}

In this issue, we publish the names of those who reviewed manuscripts for us in 2012 .

The Editor-in-Chief, Specialist Editors and everyone involved in publishing BJC would like to extend our sincere thanks to them for contributing their expertise and time. Our referees play an invaluable role ensuring that $B J C$ continues to publish the high quality original papers and reviews that make it one of the world's leading oncology journals.

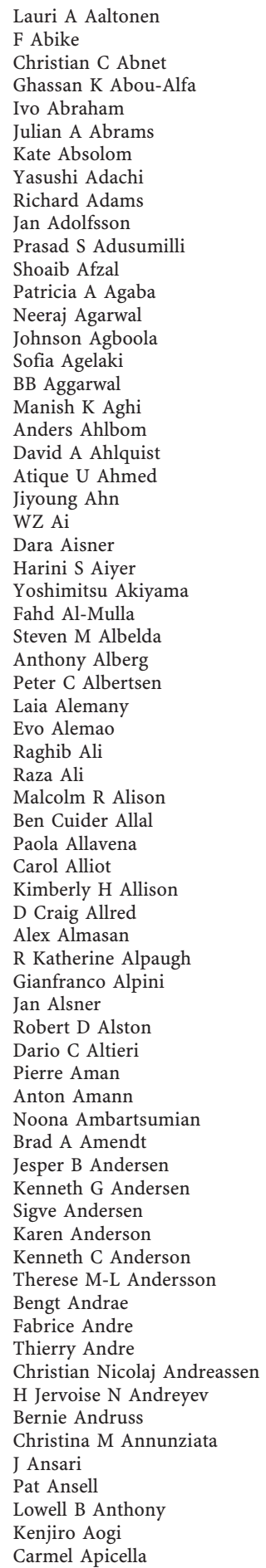

Andrea B Apolo

Yukie Araki

A Ardizzoni

Hendrik-Tobias Arkenau

Andrew J Armstrong

Rudolf Arnold

Jason D David Arroyo

JB Arterburn

Anna Arthur

Nils D Arvold

Kiyoshi Asada

Margaret Ashcroft

Irene Asmane

Rajalakshmi Asur

Djordje Atanackovic

Nadine Attal

Marc Aubry

Riccardo A Audisio

Christi K Augustine

Maria Laura Avantaggiati

AA Ayantunde

Basem Azab

Laurent Azoulay

Edouard Azzam

Amalia Azzariti

Bruno Azzarone

Peter D Baade

Sadegh Babashah

Uriel Bachrach

C Backendorf

$P$ Badiee

Sunil Badve

Duk-soo Bae

Youngmee Bae

Dominique Bagnard

BC Baguley

Helen Bailey

K Baird

Emilio Bajetta

Elizabeth Baker

Anita Balakrishnan

Arjun Balar

R Balasubramanian

Sabapathy Balasubramanian

Bo Baldetorp

Editta Baldini

Steven Balk

Fran Balkwill

Karla Ballman

Fatima Baltazar

Katarina A Balter

Cynthia Bamdad

Yung-Jue Bang

Clare Bankhead

Maggi Banning

Heike Bantel

Vickie E Baracos

Mariagnese Barbera

Rosa Björk Barkardottir

William Barlow

Mandy Barnett

Francis A Barr

Valeria Barresi

Jennifer H Barrett

Kai Bartkowiak

Rupert Bartsch

Julian Barwell

JR Basile
Bristi Basu

Andrew R Bateman

Iacopo Baussano

Jane Bayani

Harry D Bear

R Bellocco

Ittai Ben Porath

Doris M Benbrook

Virginia Benito

Idriss M Bennani-Baiti

Ian Bennett

James R Berenson

Adam C Berger

Anders Berglund

Justo Lorenzo Bermejo

PS Bernard

Luisa Bernardinelli

René Bernards

Eric J Bernhard

Edurne Berra

Franco Berrino

Donald A Berry

Paola Bertuccio

Marianne Berwick

Gyan Bhanot

Parveen Bhatti

Nirmala Bhoo Pathy

Giampaolo Bianchini

Roberto Bianco

Roy Bicknell

Pernille E Bidstrup

RJ Biggar

Ioannis Biliatis

Monica Binaschi

Brenda Birmann

Daniel Birnbaum

Gianni Bisogno

Trever Bivona

Anneke C Blackburn

Fiona Blackhall

Franz Blaes

Roman Alexander Blaheta

FE Bleeker

Ira Bleiweiss

Maria Blettner

GC Blobe

Carl Blomqvist

Mark Bloomston

K Blyth

Otto C Boerman

Julie L Boerner

Deborah A Boggs

John D Boice, Jr

Stig Egil Bojesen

Narikazu Boku

Richard Bold

Elfriede Bollschweiler

Gianni Bonadonna

A Bonetti

Arnaud Bonnomet

Petri Bono

Stefanos Bonovas

Michael A Bookman

Joachim Boos

Katherine LB Borden

Emma Borras

Josep M Borras

Silvano Bosari
Paolo Boscolo-Rizzo

Cristina Bosetti

F T Bosman

Donald Bottaro

Epie Boven

Norman F Boyd

Helen Boyle

Terry Boyle

Boudewijn Braakhuis

Chris Bradley

Filip Braet

Paloma Bragado

Kate E Brain

Karen Brajao

Elisabeth Brambilla

Vivien Bramwell

Sven Brandau

Yvonne Brandberg

Boudewijn Brans

Theodore M Brasky

Klaus Brasso

Michael Braun

Francesca Bravi

Caroline Bray

Joanna Brell

Dean E Brenner

Hermann Brenner

Emilio Bria

John Bridgewater

Maree Brinkman

Robert G Bristow

Matthias Brock

Alexander S Brodsky

Massimo Broggini

Andrew J Brown

Karen Brown

Linda Brown

Michael P Brown

Robert Brown

Adam Brufsky

Jean-Luc Brun

Nils Brunner

Richard Bucala

Trine Z Buch-Hansen

Tomas Buchler

Donald J Buchsbaum

Vishwanie Budhram-Mahadeo

Ronald M Bukowski

Frank Buntinx

Maximilian Burger

MV Burgesser

Robert D Burk

Michael Burke

Bryan Burmeister

Alan Burnett

Klaus J Busam

Martin Bushell

Gianni Bussolati

Phyllis N Butow

Zeeshan Butt

Reinhard Buttner

Marc Buyse

Giuseppe Cabibbo

Stefano Calza

David Cameron

Helen E Campbell

Ian Campbell

Neil Campbel 
M Campbell-Thompson

Kevin Camphausen

Silvana Canevari

Rosa Canuto

Liang Cao

Carlo Capella

Miguel Carballo

David P Carbon

$\mathrm{X}$ Carcopino

Robert JG Cardnell

Lisa Carey

Chiara Carlomagno

Analia Carmo

Amancio Carnero

F Carozzi

Catherine Carpenter

Norman Carr

Alfredo Carrato

Giuseppe Carruba

William E Carson

JA Carter

Jim Cassidy

Philippe Alexandre Cassier

Jorge J Castillo

Carlo Castoro

Didier Cataldo

Luca Cegolon

Paloma Cejas

James R Cerhan

Andres Cervantes

Rohit Chahal

Marc C Chamberlain

Setsuko K Chambers

June M Chan

Michael WY Chan

George J Chang

Hong Chang

Wen-Wei Chang

Jenny Chang-Claude

Stephen J Chanock

Judith-Anne W Chapman

Kellie A Charles

Jessica N Charlet

Anil K Chaturvedi

Ian Chau

Peh Yean Cheah

Dong Chen

Gong Chen

Jen-Shi Chen

Lin Chen

Wen Yong Chen

Xueyu Chen

Yao-Tseng Chen

Yingbei Chen

Georgia Chenevix-Trench

Shi-Yuan Cheng

TY Cheng

Louis Chesler

Dennis S Chi

Paolo Chieffi

EG Chiorean

Julia Clare Chisholm

Rowan T Chlebowsk

Gabriel Chodick

Haesun Cho

Kang-Yell Choi

Seungtaek Choi

Yoon-La Choi

Christos Chouaid

Toni K Choueiri

Laura Q Chow

Brock C Christensen

Quincy Chu

Michael H Chung

Joseph Ciccolini

Ashley Cimino-Matthews

Marco Ciotti

Kevin P Claffey

Christina Clarke

Robert Clarke

Mark Clements

Mark Clemons

Gary M Clifford

Steven J Cohen

Bertrand Coiffier
Henriqueta Coimbra Silva

Soren Cold

Helen Coleman

Helen G Coleman

Michel P Coleman

Helen M Coley

Laura Collins

Howard Colman

Pierre-Emmanuel Colombo

Ramon Colomer

Paolo Comoglio

Gerolama Condorell

Roisin M Connolly

Dario Consonni

Gordon Cook

Rebecca Cook

Marcus Cooke

Colin S Cooper

Matthew R Cooperberg

John A Copland

Brian L Copple

Pippa G Corrie

Laura I Cosen-Binker

Lorenzo Cosmi

Javier Cotignola

Aimee Crago

Thorsten Cramer

$\mathrm{CH}$ Crane

Jenette Creaney

Isabelle Cremer

Lucio Crino

Deirdre P Cronin-Fenton

Tom Crosby

Simon Crouch

Gabor Cserni

A Cuenda

BS Cummings

David Cunningham

Margaret Currie

Christina Curtis

Rochelle E Curtis

Jack Cuzick

Kamila Czene

Ricardo Díez Valle

Antonio B D'Assoro

Kathleen D'Hauwers

Maurizio D'Incalci

Hagit Dafni

Suzanne E Dahlberg

Bingbing Dai

Min Dai

Maria Grazia Daidone

Luigino Dal Maso

David C Dale

Amanda J Daley

Angus G Dalgleish

Bertil E Damato

DC Damin

Romano Danesi

Sarah Danson

Giovanna Danza

Sarah Darby

G Datta

Adil I Daud

Sarah E Daugherty

Catharina de Lange Davies

Michael A Davies

Ian D Davis

Scott Davis

Shaheenah Dawood

Fiona L Day

Pradip De

Enrique de Alava

Geertruida H de Bock

Loretta De Chiara

Sara De Dosso

Aimery de Gramont

Ignace de Hingh

Daphne de Jong

Michele De Laurentiis

Maria Antonietta De Loris

F De Marinis

MC De Martino

Paola De Nardi

K De Preter
Maria De Santis

Alfonso De Stefano

Chris de Wolf

L DeAngelis

Roberta DeAngelis

Jason LJ Dearling

Ralph J DeBerardinis

Waldemar Debinski

P Debourdeau

Bruno Degano

Luis Del Valle

Brett Delahunt

Laura DeLong Wood

Douglas James Demetrick

DL DeMets

David Denardo

IME Desar

Vanessa Deschoolmeester

Vincent Detours

Nandini Dey

AS Dhillon

Andrea Di Cataldo

Violante Di Donato

Marcello Di Nisio

G Di Pietro

Giuseppina Di Stefano

Eleftherios P Diamandis

Marquez-Garban Diana C

Mireia Diaz

Paul W Dickman

Francesco Dieli

Palma Dileo

Joakim Dillner

Robin M DiMatteo

Wei-Qun Ding

David Dingli

C Michael DiPersio

Luc Yves Dirix

Yves Dittmar

Hongdo Do

Sheila A Doggrell

Toshihiko Doi

Susan J Done

Jessica Donington

Peter Donnelly

Frede Donskov

Isabel dos Santos Silva

Zohar A Dotan

Q Ping Dou

Chyke A Doubeni

Jean-Yves Douillard

Mitch Dowsett

Vesna V Dragutinovic

Jennifer Drahos

George Dranitsaris

Ross Drayton

Merete Drevvatne Bugge

Melanie Drolet

Liqin Du

Xianglin $\mathrm{L} \mathrm{Du}$

Andreas du Bois

Attila Dubecz

Raymond N DuBois

Renatta Duchnowska

Arkadiusz Z Dudek

$P$ Dursun

Grace Dy

Martin Dye

Lars Dyrskjot

Agnieszka Dzikiewicz-Krawczyk

Martin M Eatock

Norman L Eberhardt

John Ebos

MA Ebrahim

Martin J Edelman

Iris E Eder

Claire Edwards

Dylan R Edwards

Ros Eeles

Friederike Egberts

Scott Eggener

Alexander MM Eggermont

Dennis A Eichenauer

Pierre Eid

Anders Ekbom

Goran Ekelund

Karin Ekstrom-Smedby

Adel El-Naggar

$S$ Elias

Heather Eliassen

Lina Eliasson

Gerda Engholm

Robert Enns

Meira Epplein

Alan L Epstein

Jonathan I Epstein

Mikael Eriksson

Serkan Erkanli

Verda Erkizan

Mark Erlander

Janine Erler

Edzard Ernst

Bernard Escudier

Ferry ALM Eskens

Marcos Estecio

DGR Evan

Jeff Evans

Nathan K Evanson

Muller Fabbri

Oluwole Fadare

Jean Faivre

Lorenzo Falchi

Gerald S Falchook

Alfredo Falcone

Jia Fan

Joachim Fandrey

Dianchun Fang

Roger Feakins

Noah Federman 
Anna Fyrberg

Yesim Gökmen-Polar Helmut Erich Gabbert

Dmitry I Gabrilovich

Giovanna Gagliardi

Georgios Gakis

JP Galanaud

Collette Galet

Gennaro Galizia

William M Gallagher

Sergio Gallegos Castorena

Carlos Maria Galmarini

Matthew Galsky

Moses Galukande

Daniel A Galvao

Montserrat Garcia-Closas

esus Garcia-Donas

Federico Garrido

Ignacio Garrido-Laguna

Rune Gartner

Anna Gasperi-Campani

Gemma Gatta

Piers Gaunt

Oliver Gautsch

Vittorio Gebbia

Samuel Gebre-Medhin

Val J Gebski

Jeanine Genkinger

Saby George

Vassilis Georgoulias

ose R Germá-Lluch

Jeffrey E Gershenwald

Andreas J Gescher

Paula Ghaneh

Rahel Ghebre

Francois Ghiringhelli

Karthik Ghosh

Attilio Giacosa

Anne Genevieve Gilg

Olivier Gires

Dilip Giri

Rosalind Given-Wilson

Laurence Gladieff

Jonathan M Gleadle

Bengt Glimelius

Stefan Gluck

Andrew K Godwin

James J Goedert

Shom Goel

John R Goffin

Elspeth Gold

Jeremy Goldharber-Fiebert

Jonathan W Goldman

Rado Goldman

Birgitte Goldschmidt Mertz

Robert N Goldstone

Ana Maria Gonzalez-Angulo

Ellen L Goode

Shaun Goodyear

Alan N Gordon

Rachel Gormley

Arko Gorter

Walter H Gotlieb

Eyal Gottlieb

Sophie Gourgou-Bourgade

Charlie Gourley

Carsten Gründke

William B Grant

Nicola M Gray

Steven G Gray

Mel Greaves

Jane Green

John A Green

Diana Greenfield

John Greenman

Vanesa Gregorc

Richard I Gregory

David Grimwade

Sergey Grivennikov

Cary P Gross

Christophe Grosset

Irene Grossmann

Karsten Grote

Thomas W Grunt

Viktor Grunwald
James L Gulley

Elisabeth Gummersbach

Andreas Gunthert

Bin Guo

Wenjun Guo

Kalpna Gupta

Ramesh C Gupta

Satish K Gupta

James G Gurney

Clement K Gwede

John BAG Haanen

Jens K Habermann

Ulrich Hacker

Jeffrey $\mathrm{H}$ Hager

Eva Haglind

John Hainsworth

Neda Haj-Hosseini

KJ Halazun

Carol Hall

Peter A Hall

Daniel G Haller

Paul Haluska

Paul Hamberg

Michael R Hamblin

Jada G Hamilton

Stanley R Hamilton

William T Hamilton

Lalle Hammarstedt

DS Han

Haiyong Han

Samir Hanash

Andrew Hanby

Torben Frøstrup Hansen

Clare Harley

Diane M Harper

Mark Harries

Michael Harrison

Mikael Hartman

Douglas J Hartmann

R Donald Harvey

A Hata

Manfred Hauben

Alexander R Haug

Richard E Hautmann

Lukas JAC Hawinkels

D Neil Hayes

John D Hayes

David W Hedley

Christopher Heeschen

Oskari Heikinheimo

Volker Heinemann

Markus M Heiss

Florian Heitz

Dan Hellberg

Samuli Helle

TO Henderson

Daniel YC Heng

Christophe Hennequin

Maria Henriksson

N Lynn Henry

Song Her

Louise Herbild

Kerstin Hermelink

Javier Hernandez-Losa

Ingrid Herr

Rolando Herrero

EM Hersch

Kenneth R Hess

Viviane Hess

Eric Hesse

Roman Heuer

Aram Hezel

Oonagh T Hickey

Chindo Hicks

Bert Hildebrandt

Richard P Hill

Lisa E Hines

Eiso Hiyama

Steven N Hochwald

M Hockel

Geir Hoff

Wayne Hofsetter

Erin Hofstatter

ME Holick

Daphne Hompes
Tetsuya Honda

Angela Hong

NJ Hong

Kanya Honoki

Nicolás Hopp

John Hopper

Jane A Hoppin

David Horst

Lisa Horvath

David Hosking

James R Howe

Anthony Howell

Robert EJ Howells

Yih-Shou Hsieh

CS Hsu

Juana Hsueh-Fen

GF $\mathrm{Hu}$

R Stephanie Huang

Sui Huang

Peter E Huber

Robert A Huddart

RS Hudson

Dennis Hughes

JW Huh

WW Huh

Laetitia Huiart

Mark A Hull

Michael Hunerbein

Keith Hunter

Amandine Hurbin

Chris Hurt

Jean A Hurteau

Herbert I Hurwitz

Iain R Hutcheson

Mark R Hutchinson

Barry Iacopetta

Nuhad Ibrahim

OK Idowu

Natalia Ignatenko

Katsunori Iijima

Hisa Iinuma

JNM Ijzermans

Daniel J Indelicato

JR Infante

Kazushi Inoue

Manami Inoue

Maki Inoue-Choi

Ralph P Insinga

Toshiyuki Ishiwata

Mitsuru Ishizuka

Mircea Ivan

Tomoo Iwakuma

Namiki Izumi

Karsten Juhl Jørgensen

Bart Jacobs

Didier Jacqmin

Martine J Jager

Sebastien Jaillon

Rakesh Jain

Anders Jakobsen

Maija Jakobsson

Janusz Jankowski

Filip Janku

Isabelle Janoueix

Maurice Jansen

Carlos Jara Sanchez

Aminah Jatoi

Gordon C Jayson

$S$ Jeganathan

Ahmedin Jemal

Alessandro Jenkner

Allan Jensen

Helena Jernstrom

Carmen Jeronimo

Yixing Jiang

Masahito Jimbo

J Jin

Moyez Jiwa

Markus Joerger

G Jogl

Bruce E Johnson

Philip J Johnson

Donna Johnston

Eric Jonasch

Jon G Jonasson

Donald JL Jones

Gareth Jones

Steve E Jones

V Craig Jordan

Elmar A Joura

Angeles Juarranz

Patricia L Judson

Claire Julian-Reynier

Barbara H Jung

Eldon R Jupe

L Kadaja-Saarepuu

Zsuzsanna Kahan

Christoph Kahlert

Thomas J Kaley

Holger Kalthoff

B Kalyanaraman

Farin Kamangar

Yae Kanai

Yukihide Kanemitsu

Yoon-Koo Kang

Steven C Kao

Henry G Kaplan

Omur Karakoyun-Celik

Amer K Karam

Hasan Karanlik

Christos S Karapetis

L Karayan-Tapon

Odysseas Kargiotis

Peeter Karihtala

Per Karlsson

Mehdi Karoui

Khosrow Kashfi

Sabine Kasimir-Bauer

Bernd Kasper 
Tish Knobf

Kevin C Knower

Sarah Kobrin

Hemant M Kocher

Marina Kochetkova

Yasuhiro Kodera

Sergio Koifman

Atsumi Kojima

E Anders Kolb

Jill Kolesar

Michael Kolodziej

Gottfried E Konecny

Koji Kono

Gerald Konrad

Kenneth Kopecky

PJ Koskinen

Rami Kotb

Gregory Kouraklis

Anita Koushik

M Krause

Irene Kriegel

Meinir Krishnasamy

Alan R Kristal

Hartmut Kristeleit

Glen Kristiansen

Guido Kroemer

M Kroiss

Kevin R Krull

M Krzystek-Korpacka

Donald W Kufe

Roland P Kuiper

Ernst Kuipers

Shalini L Kulasingam

Shaji Kumar

Hiroki Kuniyasu

Min-Liang Kuo

Peter Kuppen

K Kuroi

Anton Kutikhin

S Kuwamoto

Hau Kwaan

Natasha Kyprianou

Hedvig E Löfdahl

Matthias Löhr

Caterina AM La Porta

Carlo La Vecchi

James Lacefield

Jesper Lagergren

Pagona Lagiou

Rose Lai

Christel B Lajer

Benjamin W Lamb

Gurpreet Lamba

Daniel W Lambert

Paul C Lambert

Johnathan M Lancaster

Charles N Landen, Jr

Marene Landstrom

Sven A Lang

Ann Langius-Eklof

Ruth E Langle

Tanja Langsenlehner

Gunnar Larfors

Carlo Largiadèr

James M Larkin

Denis Larsimont

Jean-Baptiste Lattouf

Vincent Launay-Vacher

Guy Launoy

Pierre Laurent-Puig

Dominique Laurier

Malcolm Law

Wai Lun Law

Ian C Lawrance

James S Lawson

GT Layer

Andreas Lazaris

Hillard Lazarus

Eduardo Lazcano-Ponce

John Lazo

Cecile Le Page

John Le Quesne

Christophe Le Tourneau

Andrew HS Lee

Dae Ho Lee
Han Chu Lee

Jae-Lyun Lee

Jang-Ming Lee

Se-Hoon Lee

Sug Hyung Lee

Maarit K Leinonen

Marcis Leja

Riccardo Lencioni

Frances E Lennon

Suzanne Lentzsch

James E Lester

Hing Y Leung

Francis Levi

Jon D Levine

BI Levy

Wyn G Lewis

Donghui Li

HC William Li

Dong Liang

LM Liao

Michael R Lieber

Sandra Liekens

TJ Lightfoot

Marjolijn Ligtenberg

Biaoyang Lin

Hui-Kuan Lin

L Lin

Yi-Tsung Lin

Claus Lindbjerg Andersen

Annika Lindblom

Sara Linden

Stefan Linder

Barbro Kristina Linderholm

Sabine Linn

Esther H Lips

Allan Lipton

Michael P Lisanti

Saskia Litiere

Virginia R Litle

Mark Little

Liang-Ming Liu

Rui Liu

Borje Ljungberg

Josep Lloreta

Sharon Lobert

Dileep N Lobo

Tillmann Loch

David M Loeb

Richard FA Logan

Sherene Loi

Sybille Loibl

Bal L Lokeshwar

Anna E Lokshin

Hilda Lomeli

PD Loprinzi

Florian Lordick

Karen Lounsbury

Fotios Loupakis

Bo Lu

$\mathrm{H}$ Lu

Jiachun Lu

Jun Lu

Shujing Lu

Yani Lu

Yong-Jie Lu

Yunxia Lu

D M Lubman

Gary Lyman

Fiona Lyng

Georgios Lyratzopoulos

S Mølle

Jie Ma

Patrick C Ma

Qing $\mathrm{Ma}$

Georgie Macarthur

GJ Macfarlane

Helen MacKay

Yoshihiko Maehara

Davide Maggi

SM Magrini

Nupam P Mahajan

Jane Maher

Georges Maire

Markus J Makinen

Núria Malats
Reza Malekzadeh

Peter Malfertheiner

Nea Malila

Tsz-Kwong Man

Arun K Mankan

G Bruce Mann

Judith Manola

Alberto Mantovani

Lulu Mao

Juan Antonio Marchal

M Mareel

Vitaly Margulis

Pascal Mariani

Christophe Mariette

Alessandro Marinaccio

Maurie Markman

Morgan Marks

Laura AV Marlow

Christine Marosi

Rolf Marschalek

John WM Martens

Miguel Martin

Olga A Martin

Paul T Martin

Robert CG Martin

Sarah Martin

Michele Masetti

Gianluca Masi

Lorenzo Masieri

Nick Maskell

L Stewart Massad

Christophe Massard

Suleiman Massarweh

Leon F Massuger

Daniela Matei

Marie-Christine Mathieu

D Mavroudis

Deborah K Mayer

Marty W Mayo

Vera Mazurak

Sam Mbulaiteye

CJ McCabe

Ruth McCorkle

Ultan McDermott

James Ted McDonald

Ken McElreavey

Grant McFadden

Patricia M McGowan

WP McGuire

Colleen C McLaughlin

Howard L McLeod

Pamela McMahon

Donald C McMillan

Richard JQ McNally

Douglas G McNeel

Richard P McQuellon

Joshua J Meeks

Janice M Mehner

Philip Meijnen

Muhammed Ashraf Memon

Marc S Mendonc

Maxwell V Meng

Sebastiano Mercadante

Johannes HM Merks

Ray Merrill

Wells A Messersmith

Ludwine Messiaen

Jukka H Meurman

Fremonta Meyer

Guy Meyer

KL Meyer-Siegler

Raymond E Meyn

JL Meza

Saiful Miah

Michael Michael

M Dror Michaelson

Dominique S Michaud

Karin B Michel

Wolfram Miekisch

Stephen D Mikolajczyk

Linda R Mileshkin

Michael Millward

Murielle Mimeault

Hironobu Minami

John Minna

Akiva Mintz

Saverio Minucci

Olivier Mir

Elizabeth D Mitchell

Gillian Mitchel

Timur Mitin

Koji Miyahara

Hideaki Miyake

Masaru Miyazaki

Seyed Javad Moghaddam

Samuel C Mok

Tony SK Mok

Jan J Molenaar

Mecker Moller

Elin Mollerstrom

Timothy J Molloy

H Momota

Alison M Mondu

Bradley J Monk

Evelyn M Monninkhof

Joseph Monsonego

Ali Montazeri

G Monteith

Kathleen N Moore

Patrick S Moore

Meena Moran

Jerome Moreaux

Jan S Moreb

Daniel Moreira

B Morgan

Todd M Morgan

William F Morgan

Jo Morris

PG Morris 
Philipp Nuhn

Peter Nygren

Karin Nylander

Mary ER O'Brien

Kenneth J O'Byrne

Erin S O'Connor

Norma O'Donovan

David O’Malley

Takahiro Ochiya

Gregory Offerson

Simona Ognjanovic

Atsushi Ohtsu

Alicia Frances Clare Okines

Koichi Okumura

Kiyotaka Okuno

Alexander Olawaiye

Ivo A Olivotto

Olufunmilayo I Olopade

Adedayo Onitilo

Joost Oppenheim

Nobuhiko Oridate

Raymond J Osborne

B Oskooei

Michael J Overman

Ingrid Pabinger

Karel Pacak

Simon Pacey

Russell K Pachynski

Sara Pai

Frank Pajonk

Jose Palacios

Juan P Palazzo

Ignacio Palmero

Diane Palmieri

Giuseppe Palmieri

Richard Palmqvist

Edward Pan

Orestis Panagiotou

BN Pandey

Liron Pantanowitz

Klaus Pantel

Xavier Paoletti

Christos Paraskeva

Saverio Paris

Daniel Park

Jeong-Yeol Park

Se Hoon Park

Maurizio Parola

Paola Parrella

I Pastan

Sujata Patil

Julietta Patnick

Bishnuhari Paudyal

J Paul

EC Paulson

Nicholas Pavlidis

Yudi Pawitan

Mick Peake

Loretta L Pecchioni

Rémy Pedeux

Marc Peeters

Anthony Pegg

Claudio Pelucchi

Luiz O Penalva

Nicolas Penel

Xinsheng Peng

George Pentheroudakis

Edith A Perez

Rodrigo O Perez

Sonia Pernas

Thomas V Perneger

Francesco Perrone

Danilo Perrott

Antoinette Perry

Godefridus J Peters

SH Petersen

Brenda Petrella

Andreas Pettersson

Raffaele Pezzilli

Annalisa Pezzolo

Paul DP Pharoah

Darcy Phelan

Philip A Philip

Kelly-Anne Phillips

Wayne A Phillips
Claude Pichard

Martin Pichler

Jean-Yves Pierga

Blanchard PIERRE

M Pillai

Dietmar Pils

Pedro Pimentel-Nunes

Pascal Pineau

Michael Pinkawa

Alexandros Pintzas

Edyta Pirog

Giuseppe Pirozzi

Paola Pisani

Valerie Pittet

E Pizzo

Elizabeth R Plimack

Alida J Pokoradi

Jerry Polesel

Giuseppe Poli

Bernhard M Polzer

Sanjay Popat

IS Poritz

Tyrone Porter

Nancy Potischman

Anne-Lise Pouliquen

Don W Powell

Scott Powers

Setia Pramana

PK Prasad

Vidudala VTS Prasad

Aleix Prat

Jochen HM Prehn

Malin Prenkert

John N Primrose

J Howard Pringle

Catrin Pritchard

Prathima Prodduturi

Xose S Puente

Eero Pukkala

MH Qazilbash

C Quantin

Denis Querleu

Gwen Quinn

Philip Quirke

Françoise Rédini

Kathrine Røe

Brigitte Rack

Junaid Rafi

Massimo Raimondo

Emad A Rakha

Pranela Rameshwar

Maria Ramos

Torbjorn Ramqvist

Pawan Randev

Elena Ranieri

Malcolm Ranson

Sheela Rao

Julie Ratcliffe

Susanne Rautiainen

Isabelle Ray-Coquard

Eric Raymond

Albiruni RA Razak

Ruth Rechis

Anne Hansen Ree

Nicholas Simon Reed

Colin J Rees

Peter Reichardt

Alison Reid

Mary E Reid

Anke C Reinacher-Schick

Monica Reinholz

Niels Reinmuth

Scot C Remick

Jordi Remon

Huan Ren

Daniel J Renouf

Peggy Reynolds

Sir Mike Richards

Christopher Ricketts

Frederick R Rickles

Herbert Riechelmann

Sabine Riethdorf

David Rimm

Brian I Rini

Robert T Ripley
Gail Risbridger

Olivier Rixe

Kathryn Robb

David W Roberts

Sally Roberts

Kim Robien

Mark E Robson

Andrea Rocca

Andrew W Roddam

$\mathrm{R}$ Roesler

Mark S Roh

Sabine Rohrmann

Eve Roman

Massimo Romani

Alvaro Ronco

Jeanine Roodhart

Matti A Rookus

Rafael Rosell

Lee S Rosen

Matteo Rota

Marianne G Rots

Elizabeth Ann Roundhill

Campbell SD Roxburgh

Laura Rozek

Volker Rudat

Kathryn J Ruddy

EH Ruder

Charles M Rudin

SJ Russell

Antonio Russo

Anil K Rustgi

Piotr Rutkowski

Lisa Ryden

Steve Ryder

W David J Ryder

Ricardo Sánchez-Prieto

Fred Saad

JM Saez

Vikrant V Sahasrabuddhe

Nagahiro Saijo

Pierre Saintigny

Hideyuki Saito

Antti Sajantila

T Sakmar

Ritu Salan

Flavio Salazar-Onfray

Marcela Salomao

Andrea Salonia

Talya Salz

E Samalin

Sven Sandin

R Sankaranarayanan

Alessandro D Santin

Armando Santoro

Anna Sapino

Diana Sarfati

Fazlul H Sarkar

Hervé Sartelet

Michael B Sawyer

Philip J Saylor

Peter T Scardino

Mario Scartozzi

Michael Schaapveld

Beat Schaefer

TJ Schall

Jorn Markus Schattenberg

Georgios Scheiner-Bobis

Silke Schelenz

Howard I Scher

Marco Schiariti

WP Schiemann

Mark Schiffman

Reinier O Schlingemann

Fernando Schmitt

Gunter Schneider

Minouk J Schoemaker

Jonathan Gregory Schoenecker

Patrick Schoffsk

John H Scholefield

Katrine R Schonnemann

Mario Schootman

Leslie R Schover

Peter Schraml

Jochen Schubert

Kai Schuh

HM Schuller

Joachim Schuz

Marc D Schwartz

Simo Schwartz Jr

Lee S Schwartzberg

Michal R Schweiger

Kieran Scott

Peter Sedlmayr

Josefin Segelman

Christof Seidl

Harold E Seifried

Tanguy Seiwert

Naohiko Seki

Thomas J Semrad

Tan Chuen Seng

Diego Serraino

Raguel Seruca

Mukund Seshadri

Gautam Sethi

Maida J Sewitch

Sana Sfar

Kashif Shafique

Yuval Shaked

Masood Shammas

C F Sharpley

Patricia M Sheean

Jing Shen

Wei Shen

Xi-Zhong Shen

Katherine Sherba

Jian-Guo Shi

Lei Shi

Qian Shi

Cleveland G Shields

Ie-Ming Shih 
Gunnar Steineck

E Steliarova-Foucher

Sally Stenning

Arnulf Stenzl

Olof Stephansson

Howard Stern

Richard G Stevens

Clinton Stewart

Douglas A Stewart

Bangyan Stiles

Martin R Stockler

MR Stolley

Rachael Stolzenberg-Solomon

Jennifer Stone

Britta Stordal

Alexander J Stratigos

Berthold Streubel

Reiner Strick

Carrie Stricker

Euan Stronach

Til Sturmer

Nazareno Suardi

K Subbaramaiah

Shi-Yong Sun

Yi Sun

Lillian Sung

Sripathi M Sureban

Robert L Sutherland

Helena Svensson

WE Svensson

John Swanson

Charles Swanton

Colleen Sweeney

Anthony J Swerdlow

Elizabeth Swisher

R Paul Symonds

Konstantinos N Syrigos

Attila Szabo

Zoltan Szallas

Tibor Szarvas

Peter Szlosarek

Dennis Taaffe

Lazlo Tabar

Scott T Tagawa

Sharareh Taghipour

Tetsuya Taguchi

M Takahashi

Toru Takano

Hiroyuki Takei

Robert Takes

Akinobu Taketomi

Yuichi Takiguch

Takahisa Takino

Mats Talback

Giovanni Tallini

Rulla M Tamimi

Marco Tampellini

Winston W Tan

Naping Tang

Ian F Tannock

Janos Tanyi

Jolanta Tarasiuk

Doug Taupin

Alessandra Tavani

Philip R Taylor

Niall Christopher Tebbutt

Manuel R Teixeira

Sarah Temkin

Paul Tempst

Ioannis Tentes

William P Tew

Johannes Thaler
Theresia Thalhammer

GN Thalmann

Armin Thelen

Dan Theodorescu

Brigitte L Theriault

Chrissie Thirlwell

Anne Thomas

Seufferlein Thomas

Alastair M Thompson

Mangesh Thorat

Peter Thuss-Patience

Madeleine MA Tilanus-Linthorst

Andrea Tinelli

Marc Tischkowitz

Arthur S Tischler

Andrew Titman

Kjersti Tjensvoll

Marc Tjwa

Aaron Tobian

Giuseppe Toffoli

Ladislav Tomasek

G Tomasello

Erika Toth

David Tougeron

Nham Tran

Phuoc T Tran

Lois B Travis

Ruth Travis

Mary Lynn Trawick

Tom Treasure

Lyndal Trevena

Dimitrios Trichopoulos

EL Trimble

Angela D Trobaugh-Lotrario

Arne Trummer

Chi-Neu Tsai

Chantal Tse

Apostolia-Maria Tsimberidou

Nikolaos Tsoukalas

Pelagia G Tsoutsou

Tohru Tsujimura

E Tulchinsky

James Turkson

Robin Turner

Chris Twelves

V Tzelepi

Hideki Ueno

Kimio Ushijima

Ulka Vaishampayan

Ulrich Valcourt

Patricia C Valery

Manuel Valladares-Ayerbes

Juan W Valle

Francois M Vallette

Ben Van Calster

Cornelis JH van de Velde

Marleen van den Berg

Gabri van der Pluijm

AE van der Pool

Hans J van der Vliet

Jos van Dijck

Albertus N van Geel

Dirk $\mathrm{C}$ van Gent

Carla H van Gil

FJ van Kemenade

Steven I Van Laere

Cornelis van Noorden

Edwin Van Wijngaarden

Tyler VanderWeele

Erik Vassella

Lars J Vatten

Peter Vedsted
Marcial Velasco Garrido

E Vellenga

Robert Veltri

Franco M Venanzi

Balaji Venugopal

Jasper Verguts

Johanna Verhulst

Gontran Verset

V Verwaal

Andrew Vickers

Simone N Vigod

Esther Vilaprinyo

Adam Christian Vilmar

Mark Vincen

Bruno Vincenzi

Jesús Vioque

Beth Virnig

Jamboor K Vishwanath

Andrea Vodermaier

Marco Volante

Angelika M Vollmar

Margaret von Mehren

Dietrich von Schweinitz

Adri C Voogd

Elena Voronov

Oswald Wagner

Simon Wagner

Heather A Wakelee

Ori Wald

Jo Waller

Matthew G Wallis

Craig Walsh

Tom Walsh

Roland Walter

Andrew CA Wan

Jaw-Yuan Wang

Ji Ming Wang

Tian-Li Wang

Timothy Wang

Wei-Shu Wang

Wenyi Wang

Won-Bo Wang

Zhaoming Wang

Jane Wardle

Paul Waring

Ellen Warner

Graham Warren

K Watabe

David Watkins

Eila Watson

Penelope Webb

Jeffrey Weber

Johanne I Weberpals

Britta Weigelt

$\mathrm{J}$ Weis

$S$ Weiss-Smith

Karin Welén

Claudia Wellbrock

David P Weller

Wolfgang Weyers

Stefan Wiehr

Gareth Haydn Williams

Anna-Lise Williamson

Stefan Wilop

Jacque Wils

Kathryn M Wilson

KS Wilson

Robert H Wiltrout

Heidi Wirtz

Isaac Witz

Thomas E Witzig

Dominik Wodarz
Kathleen Y Wolin

Grace Lai-Hung Wong

Sandra L Wong

Laura M Woods

Michael J Worley, Jr

Jason D Wright

Guojun Wu

Kou-Juey Wu

Yi-Long Wu

Yong-Bing Xiang

Huasheng Xiao

Qian Xiao

Qian Xie

Wei Xie

Liang Xu

$\mathrm{XX} \mathrm{Xu}$

Akira Yamada

Hiroharu Yamashita

Keishi Yamashita

Cong Yan

Tristan D Yan

Chengfeng Yang

Ling Yang

Xiaohe Yang

Qizhi Yao

Christina Yap

Timothy A Yap

John Yarnold

Weimin Ye

Winnie Yeo

William SB Yeung

Min Yi

Junji Yodo

Hiroshi Yokozaki

Kazuhiro Yoshida

Hirofumi Yoshino

Suzuki Yoshiyuki

Robin J Young

Dihua Yu

Evan Yu

Jau-Song Yu

Onchee Yu

Jian-Min Yuan

Takeshi Yuasa

Stergios Zacharoulis

S Yousuf Zafar

Ian S Zagon

Gerard Zambetti

Alfeu Zanotto-Filho

Renata Zaucha

Anne Zeleniuch-Jacquotte

Michael Zeschnigk

A L Zhang

Li Zhang

Rong-Xin Zhang

Wei Zhang

Xiuging Zhang

Xuehong Zhang

YaShuang Zhaoa

Shu Zheng

Haizhen Zhong

Kangmin Zhu

Regina Ziegler

Volker Ziller

Wolfgang Zimmerman

Teresa A Zimmers

Dov Zipori

Stanley Zucker

István Zupkó

Amado Zuri 